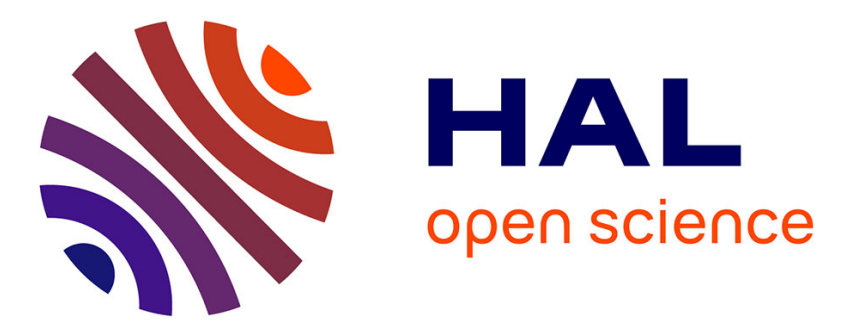

\title{
Human Heuristics for a Team of Mobile Robots
}

Charles Tijus, Nicolas Bredeche, Yves Kodratoff, Mary Felkin, Cedric

Hartland, Elisabetta Zibetti, Vincent Besson

\section{To cite this version:}

Charles Tijus, Nicolas Bredeche, Yves Kodratoff, Mary Felkin, Cedric Hartland, et al.. Human Heuristics for a Team of Mobile Robots. IEEE International Conference on Research, Innovation and Vision for the Future, Mar 2007, Hanoi, Vietnam. pp.122-129. inria-00175523

\section{HAL Id: inria-00175523 \\ https://hal.inria.fr/inria-00175523}

Submitted on 28 Sep 2007

HAL is a multi-disciplinary open access archive for the deposit and dissemination of scientific research documents, whether they are published or not. The documents may come from teaching and research institutions in France or abroad, or from public or private research centers.
L'archive ouverte pluridisciplinaire HAL, est destinée au dépôt et à la diffusion de documents scientifiques de niveau recherche, publiés ou non, émanant des établissements d'enseignement et de recherche français ou étrangers, des laboratoires publics ou privés. 


\title{
Human Heuristics for a Team of Mobile Robots
}

\author{
Charles Tijus, Nicolas Bredeche, Yves Kodratoff, Mary Felkin, Cedrick Hartland, Elisabetta Zibetti, \\ and Vincent Besson
}

\begin{abstract}
This paper is at the crossroad of Cognitive Psychology and AI Robotics. It reports a cross-disciplinary project concerned about implementing human heuristics within autonomous mobile robots. In the following, we address the problem of relying on human-based heuristics to endow a group of mobile robots with the ability to solve problems such as target finding in a labyrinth. Such heuristics may provide an efficient way to explore the environment and to decompose a complex problem into subtasks for which specific heuristics are efficient. We first present a set of experiments conducted with group of humans looking for a target with limited sensing capabilities solving. Then we describe the heuristics extracted from the observation and analysis of their behavior. Finally we implemented these heuristics within khepera-like autonomous mobile robots facing the same tasks. We show that the control architecture can be experimentally validated to some extent thanks to this approach.
\end{abstract}

Index Terms - Cognition, Autonomous Robotics, Humancentered approach, Heuristics, Multi-agents Problem Solving.

\section{INTRODUCTION}

$\mathrm{H}$ UMAN supervised learning, through imitation, instruction and guidance is a key issue in Cognitive Robotics. Our research goal is to find meaningful descriptors of human behavior, allowing a controller implementation for mimicking different human strategies, in a human-centered approach while avoiding the correspondence problem between humans and Robots.

Firstly, we present the human centered approach to Robotics and, secondly, the collection of data from humans that mimic Robots in the sense that they would have the same perceptive limitations (unknown environment, blind and using their arms to explore the environment, ...). Thirdly, we show how the human reasoning (thinking, anticipating and planning) could be used to endow robots with efficient

Manuscript received October 25, 2006. This work was supported in part by the CNRS Traitement des connaissances, apprentissage et NTIC (TCAN) and the Pascal Network of Excellence.

C. Tijus is affiliated with the Complex System Modelling and Cognition, European Joint Research Lab, Eurocontrol-EPHE and with the Laboratoire Cognition \& Usages, Université Paris 8, 2 rue de la Liberté, 93526 St Denis cedex 02 (phone: 331 49406489; fax: 331 49406754; e-mail: tijus@univparis8.fr).

N. Bredeche, Y. Kodratoff, M. Felkin and C. Hartland are affiliated with the Laboratoire de Recherche en Informatique, LRI, bâtiment 490 Université Paris-Sud F-91405 - ORSAY (e-mail: Cedric.Hartland@lri.fr).

E. Zibetti (Elisabetta.Zibetti@univ-paris8.fr) and V. Besson (over_vince@hotmail.com) are affiliated with the Laboratoire Cognition \& Usages, Université Paris 8, 2 rue de la Liberté, F-93526 St Denis. controllers.

Reaching a long-term goal in an unknown environment, that is a dynamic environment, because of the presence of other agents, would need a representation of the environment as an internal model and would need planning in order to decide upon a specific sequence of actions. However, planbased systems often break down because when the environment changes, it is difficult to maintain an accurate internal model and actions become outdated [1]. On the contrary, the adaptive-behavior approach does not follow the common theory in cognitive psychology of making extensive inferences based on internal models. Instead, it considers that an organism's response to the circumstances in its immediate environment is based on a set of rules of sensory-motor couplings (SMCs), using only the internal representations of that part of the environment currently relevant for reaching the goal. The organism is situated in its environment and it is able to independently sense and respond to the environment through its sensory receptors and effectors. Consequently, as the organism is in constant interaction with its environment through a set of SMCs, a global representation of the environment is not necessary, because the environment is its own best model [2]. In fact, only a small part of an agent's environment is relevant when it comes to taking action: the part concerning the task the agent has to perform.

In order to reach a long-term goal, according to the adaptive-behavior approach (see [2], [3], [4], [5]), it is not necessary to have previously defined plans detailing the different steps an organism can perform. Depending on the goal in question, we understand that the organism's direct contact with the environment through its sensors and effectors enables it to exploit the properties of the current state of the environment by following SMC-based rules such as "If you detect an obstacle in your way, turn right." In short, SMCs do not specify an internal representation of the environment or give a sequence of steps that must be followed to reach a goal. Instead, they specify a local-categorization process that guides the organism's relationship with the objects and other organisms in its environment.

\section{THE HUMAN CENTERED APPROACH TO ROBOTICS}

SMCs can be made through Bayesian analysis of regularities found in the environment using a complex Distributed Adaptive control requiring thousands of examples [6], without being sure that what is learnt can be properly generalized to other types of environment. SMCs could also be learnt through human guidance, by imitation [11], or developmental robotics [7]. We report our work on human 
guidance of robots through human instruction coupled with data mining successful behavioral patterns.

\section{A. The human adaptive behavior}

The human centered approach to robotics (HUCAR) we adopted is based on human instruction. We argue that human adaptation is based on categorization (see [8] for a review) and that effective instruction is to transmit categories. Categorization involves internally storing and differentiating the information we receive from our environment. When sensory receptors detect the presence of an object in the perceptual field, categorization takes place in several phases, as summarized by [9]. Firstly, a structural description of the object is made containing information on the object's most basic features (e.g. horizontal and vertical lines); secondly, the categorical representations most similar to the structural description of the detected object are selected from memory (e.g. if the object is an obstacle, the categories of objects that can be obstacles are selected, as they are all functionally and structurally similar to the current obstacle object, although the most similar category will be the one finally selected) in order to find out the proper action; and thirdly, inferences about the object are made based on the category selected (e.g. the obstacle is to be pushed).

For implementation, categorization can be seen as a set of behavioral rules or SMCs that link the sensation directly to the action and allow the organism to group and differentiate the entities in its environment and perform a specific action based on the physical properties of the entity detected. Thus, on one hand, if the organism behaves in the same way ("motor") when faced with two objects, A and B ("sensory"), we can infer that both objects are grouped in the same category. If, on the other hand, the organism behaves in a different way, we can infer that the objects belong to different categories. These grouping and differentiating mechanisms, which begin with categorization, do not need to be fully internally related. In robotics, this is called a functional definition of an object. For example, saying that "a chair is something on which someone can sit" is a functional definition of a chair. In addition, whether or not an organism behaves in the same or a different way with respect to objects depends not only on the objects' physical properties, but above all on the task the organism has to perform at a given time. The key information required to perform a given task in a given environment will influence what is learned about the environment [19]. Although the objects offer all the physical and functional properties they are identified with, it is the properties that are useful for performing the task in question that are focused on. Of all the available objects in the environment, only those with relevant properties for the task to be performed are of interest. For example, we modify the SMCs with respect to a chair depending on whether we want to sit down on it or move it from one place to another, so the SMCs are established in accordance with a property of the chair we consider to be important for the task at hand. Thus, although the properties of the object remain the same, the relevance of one of the properties (such as the property the organism uses to establish the SMC) changes according to the task in question. There is no need to be aware of all the possible objects in the environment or of all the properties that define the objects we are interested in. Of all the possible properties, the property that makes the object relevant for performing a certain task is represented locally. That is why we model categorization as a local process. This local categorization can be seen as being realized by a set of hierarchical sets of controllers.

\section{B. A three Steps Method}

This study is about how a team of three robots could behave when given the task of reaching a long-term goal, i.e. reaching an object beyond the organism's immediate spatial and temporal range.

A first approach tends to formulate the problem as the implementation of an optimal function of control. Limits of this approach are known: it stands on assumptions of reliabilities of the sensors and effectors. Moreover, the design of such algorithms is often an expensive task with limited reuse due.

A second approach is based on machine learning techniques and optimization. Within this framework, the objective is to develop algorithms making it possible to learn a driving task given starting examples (traces in a file, demonstration in real time by human, etc. - the possibilities are multiple). Several approaches are considered according to the task: locomotion (legged robot), handling (arm robot), etc. Indeed, if one can relatively easily obtain many examples of vehicle driving for the training of the locomotion [10], it is difficult to ask a human experimenter to show thousands of times the gestures to be imitated [11].

The problem at hand is to implement human-like actions according to a long-term goal. Three types of human actions are distinguished in the literature [12]: simple body actions as primitive (handling, shaking hands, ...) [13], primitive pattern activities (walking, running, that are actions that are directly perceptible and do not necessarily require interaction with other objects to be interpreted as actions [14], and goaldirected actions. Simple body actions and patterns activities executed by some actors are easily recognizable independent of context. Contrary to simple body actions and to primitives pattern activities, goal-directed actions are long time running pieces of complex actions done to reach an unseen goal. Note that pattern activities (walking) is made of simple body actions (moving legs) and that the perception of a pattern activity is complete with no missing elements. A goal directed-action is made of pattern of activities (turn left while walking) that are made of body actions (moving legs to turn left). But, contrary to pattern of activities, the perception of a goal-directed action is incomplete: why turning left for a given goal is an unseen element of the action. Thus, when humans are perceiving goaldirected actions (turning left to take the shortest way), they are making interpretations on the basis of what they are given from the physical world and of the knowledge they possess about it. In other words goal-directed actions have a richer psychological structure than simply body actions and pattern 
of activities [15]. Thus, the problem at hand is to find the super ordinate tactics and strategies that control the current goal-directed action.

Then, state of the arts in robotics hardly matches the kind of abilities people develops when performing tasks. Task execution by humans comprehends thinking, planning and anticipating [16]. Using strategies, tactics and heuristics that have semantic components acquired from the development of knowledge in semantic memory all along the lifespan. People do verbally express how and why they proceed until the specific motor actions that are under the control of automatic behavior [8], for human cognitive psychology. These two kinds of human know-how should be a guideline for Robot mimicking Humans, then generalizing.

Lets suppose an adult teaching a child, for instance how to get some bread from the bakery using a bicycle (sorry for the obvious example): the adult would provide the child with some knowledge through instruction about how to go there, with a list of subgoals, alternative subgoals, strategies and heuristics conditioned by objects features (if the first bakery is closed, if the main road is too cloudy, and so on), and would also have to give the child practical advice about how to drive the bicycle.

We reasoned that a two steps method would be the appropriate way for Human-to-Robot learning.

This is the whole Human Centered approach to Robotics that we justify as follows. Let's suppose that a task is to be done by a child. The adult that wants to explain how to perform this task will put himself at the child level [16], [17]. This is the first step of the Human Centered Approach (HCA) to robotics: to limit the perceptions of the human (e.g. with a blindfold) to find out how the human would solve robotics tasks if he only had the robot's limited sensor capacity, and which strategies, tactics and heuristic he would implement to reach the goal. This first step also solves half of the correspondence problem [13]: the human demonstrator being at the level of the robot.

The second step is the hierarchical sets of controllers, based on an extension of the subsomption control architecture [2]. We expect to find discriminative behavior for same motor actions that are under the constraints of different strategies, tactics or heuristics through the application of data mining algorithms on the behavioral data collected in step 1 and in step 2. Theses discriminative associative links will be used for the parameters of the controllers.

\section{HuMANS THAT MIMIC ROBOTS}

Because Kheperas, the target robots, have limited perception capacities ( 8 Infra-red proximity and ambient light sensors with up to $100 \mathrm{~mm}$ range), humans were asked to perform a goal-oriented task with the same kind of limitations.

We reasoned that blind people using their arms as sensors would be in quite kheperas like situations. Volunteers were given the task of finding a bottle of water in an unknown environment that was a kind of labyrinth room (figure 1): a room with tables with different orientations.

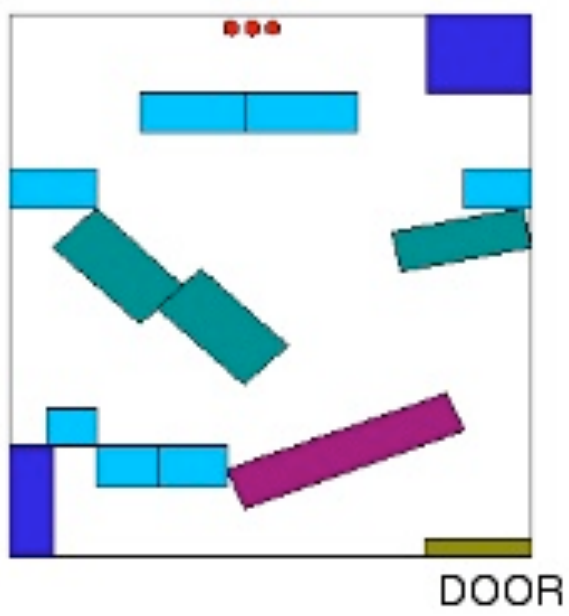

Fig. 1. An example of the search environment that is a room with tables (rectangles) of various orientations as the environment for humans that mimic robots, having the task to find bottles of water (the three dots).

\section{A. Method}

Participants were 27 subjects recruited at the Cité des Sciences et de l'Industrie de la Villette (Paris) and at the University of Paris 8 . There were grouped in order to form nine teams of three individuals.

Materials for observation were the room (at the Laboratoire des Usages en Technologies d'Information Numérique, LUTIN, at the Cité des Sciences et de l'Industrie de la Villette (Paris), tables in various orientations, bottles of water, a scarf and a headphone by participant to mask the eyes and two cameras with a tripod.

The procedure consisted in informing the 3 participants of a team that they would have to find bottles of water in the room without visual perception and without the use of vocalization or audition, and that they have to cooperate to find one bottle (for 5 of the 9 teams) or to compete to find three bottles (for 4 of the 9 teams).

Participants were also instructed that once they found the water they would have to go back to the door where the experimenter would be standing. The whole 9 sessions of searching for the water were video recorded. The videos were analyzed in order to find out how the participant proceeded to reach their goal. After the search session, each of the participants was also asked to draw the room with localization of its contents.

\section{B. Results}

Results are that all the teams found the bottles in less than 6 minutes, except for two members in a competition team that spent more than 15 minutes. The first participant of a team to find the bottles reached this goal in less than 3 minutes when participants were cooperating and in more than 3 minutes when participants were competing. Figure 2 shows two participants cooperating. 


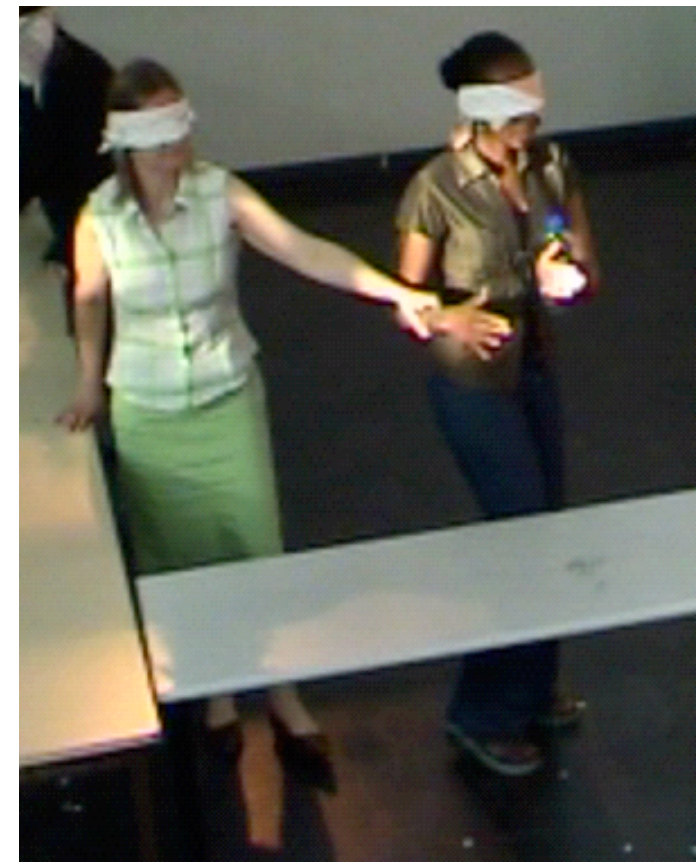

Fig. 2. Two robot-like participants that are cooperating in order to find the search target (a bottle of water).

Another result was that the drawings of the room, after the water had been found, were somewhat realistic, showing that the participants were using visual imagery, although not extensively, while searching for the target (Figure 3).

Other results are first that participants have body actions (arms, feet) to explore their close environment extensively. This means that they occupy the enclosed space in such a way that if the target were there, they would find it (figure 4-a). The largest exploration of the enclosed space has also the effect of covering the whole space of the room while having pattern activities. A behavior that was not observed in backward pattern of activities, described below.

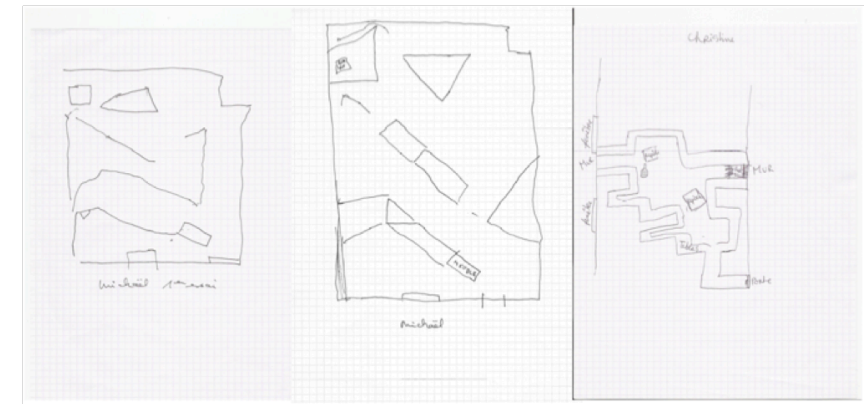

Fig. 3. Drawings of the room of figure 1 made by participants after the water search session.

The most typical primitive pattern activity was to follow the walls and the sides of the table, always being in contact (figure 4-b). This pattern of activity permits the largest exploration with body actions, while having a direction provided by the wall or the tables. This is observed in forward pattern of activities. When being in a dead end, or when having found the target, participants have forward activities for which they still have contact with the walls or tables but they do not have in this case the body actions of exploring the enclosed space.
Another typical primitive pattern activity was traveling across empty space leaving a wall or a table for another wall or table. As shown in figure 5, this is done from the corner of the current wall or table after the extensive exploration. In addition, traveling across empty space was done by well performing agents by taking a diagonal direction respectively to the side of the wall or table they were leaving.

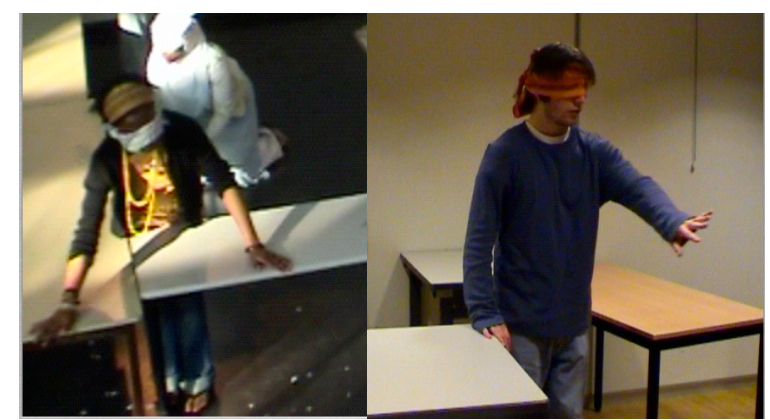

Fig. 4. (a) An example of a large exploration of the close environment with body movements, (b) a typical pattern activity that consists of following obstacles (walls and tables sides) while searching the target.
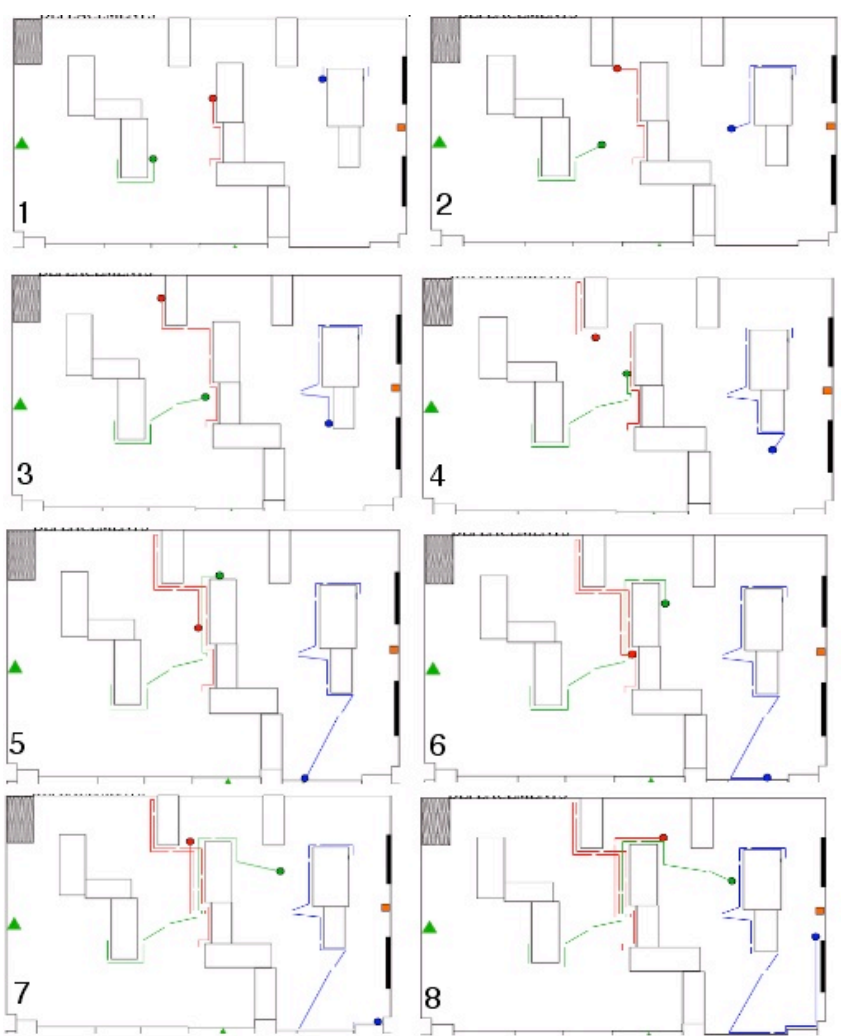

Fig. 5. From image 1 to 8 , height moves done by three cooperating human agents displaying the "following wall and sides of tables" and the "traveling space" pattern activities. From left to right in figure 1, are agents A, B and C. Agent A travels space just after a corner, Agent B and C leave the current table at the corner. Traveling space is optimized by Agent A and C (diagonal). Agent B that was the less performing shows forward "following table" (images 4, 5, 6, 7). Agent $\mathrm{C}$ is going to find the target (image 8).

Video analysis of performing behavior shows some goal directed actions that can be seen as heuristics. First was "Keep a main direction" (a west-East direction for Agent A, a NorthSouth Direction for Agent C in figure 5). Second was "avoid coming back, except in dead end": this permits to reduce the 
unexplored part of the room. Third was to take another direction when coming back. Fourth was, in case of cooperation, to take another direction than the direction taken by another agent (do not follow an Agent). Fifth was to avoid going somewhere being explored by another agent (in images 2 and 3 of figure 5, Agent $\mathrm{C}$ stops a traveling space). Sixth was to avoid repeating the same pathway. The whole set of heuristics for goal directed actions can be seen as on line planning built on an increasing internal description of the room (figure 3). Participants quickly solved the problem, except in few cases. Note that the problem could remain unsolved for agents that would have a random exploration of the room.

The search behavior displaying by agents $\mathrm{A}$ and $\mathrm{C}$ are the kind of behavior we plan to implement in virtual agents and in Kheperas through hierarchical sets of controllers.

\section{DATA MINING HUMAN BEHAVIORAL PATTERNS}

From the videos recorded by humans looking for bottles, 13 databases were created, each describing a path followed by a person in a maze. An example in these databases is a description of the situation of one person in the maze at a given time step. The description is composed of 50 "observables", meaning values that can be observed directly from the video. Examples of observables are the number of the time step, the $(\mathrm{X}, \mathrm{Y})$ coordinates of the body, the $(\mathrm{X}, \mathrm{Y})$ coordinates of each hand, the orientation of the body, etc. A simulator called "Maze" was specifically implemented to help automate this data collection (cf. fig. 6). Through the use of Maze, the videos were translated into tables of raw data where each column was an observable and each row a time step (4 time steps by second of video). The class value to be predicted was an expert-given appreciation of the type of strategy the person applied at that time step (e.g. random walk, follow wall, explore surface, etc.).

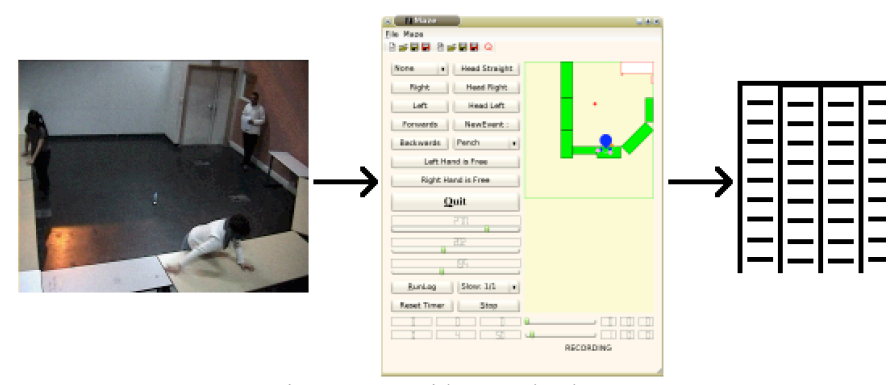

Fig. 6. From videos to database.

From these observables, "primitives" were built. Primitives are what in data mining are generally referred to as attributes. They result from a preprocessing of the raw observable data.

The need for such preprocessing immediately becomes apparent if we think of the time step observable. Classification algorithms are opportunistic in the sense that they could, for example, build their model of the data by noticing that between time step $\mathrm{X}$ and time step $\mathrm{X}+\mathrm{T}$ the human agent which actions are described in the database perform random exploration, leading to a true but useless observation with regards to a relevant model of what is a random exploration and when it takes place (i.e. the environmental stimuli and speed of movements are much more important that time tags).

Other primitives are less straightforward to determine. One of such is "KNOW(OBST)" which means, for all obstacles OBST and at every time step, whether the person in the maze has already encountered about that particular obstacle or not. The value of that primitive is zero for all obstacles when a person enters a maze, and is one, for a given obstacle, when the person is directly touching that obstacle. But how should it behave once the person breaks contact with the obstacle? It is obvious that people in the maze remember previously encountered obstacles, and that this memory plays a part in their behavior. For example, a person might walk off a little way into empty space and then come back to touch the table he started from. This is deliberate, it is part of that person's strategy, so the value of $\mathrm{KNOW}(\mathrm{OBST})$ for that obstacle cannot be set back to zero right at the moment when the physical contact is broken. After a while, though, as a person wanders through the maze, they forget where this obstacle is relatively to them: they can bump into it. Or, when they again come into contact with it, they can explore its surface as they would explore a previously unexplored surface. So the value of KNOW(OBST) must be set back to zero (or very near zero) before the time at which a new contact with this obstacle would lead to a second exploration of it. The value of KNOW(OBST) must then decrease according to some distance function, for example using the sigmoid like function (smooth transition through time) - in such a case there are still parameters to determine : (1) the "hold" period during which the occurrence is still in memory and (2) the steepness of the curve in the sigmoid function (smoothness of the transition towards forgetting). Our method for setting these parameters is basically to offer to our classification algorithms several versions of the same primitive and to let the classification algorithm choose. Another way, more time-consuming, is to run the algorithm several times, once for all possible version of the primitive we are testing, and to select the version from which the classifier built the best model according to cross validation across several databases. Some classification algorithms are easier to tune in this way than others. Naive Bayes, which assumes attribute independence, is the easiest, but would be useless for our purpose because it does not build an understandable model.

We rely on C4.5 learning algorithm [20] because of its widely-known efficiency and because it builds models of the underlying distribution of class values according to attribute values which can be read and understood even by a non-expert human and which can easily lead to robot controller implementation. The drawback is that with $\mathrm{C} 4.5$ one-by-one primitive function selection is not guaranteed to produce the best results because version $\mathrm{v} 1$ of primitive $\mathrm{P}$ may be best in some context while version v2 will be best in another context, for example in the context which will occur after the next primitive has been tuned. Often, though, the simplest 
formulations of the primitives are the best. It appears that one of the best indicators in terms of movement is simply the binary variable indicating whether the person in the maze has moved or not since last time step. In retrospect, looking at the decision tree induced by $\mathrm{C} 4.5$, it becomes clear that this single primitive goes a long way into differentiating the class "No exploration" from the other classes taken together.

Once selected, the primitives are grouped and arranged into tactics. Tactics are context-dependent descriptions of movement that can be implemented in a robot controller. Tactics include search tactics (table top exploration, ground exploration, etc), movement tactics (following a wall, following a table, going across empty space in approximately a straight line, etc), following an obstacle tactics (with one hand, with two hands, etc) and obstacle detection tactics (with one hand, with two hands, bump, etc). Aside from this exact modeling of behavior onto a robot, another indirect use of the results provided here is helping the human programmer for designing robot adaptive control architecture for solving similar problems (see next section).

One should note that an extension and ongoing work of this work is about data mining time series to extract behavioral patterns.

\section{RoBOt IMPLEMENTATIONS OF HUMAN HEURISTICS}

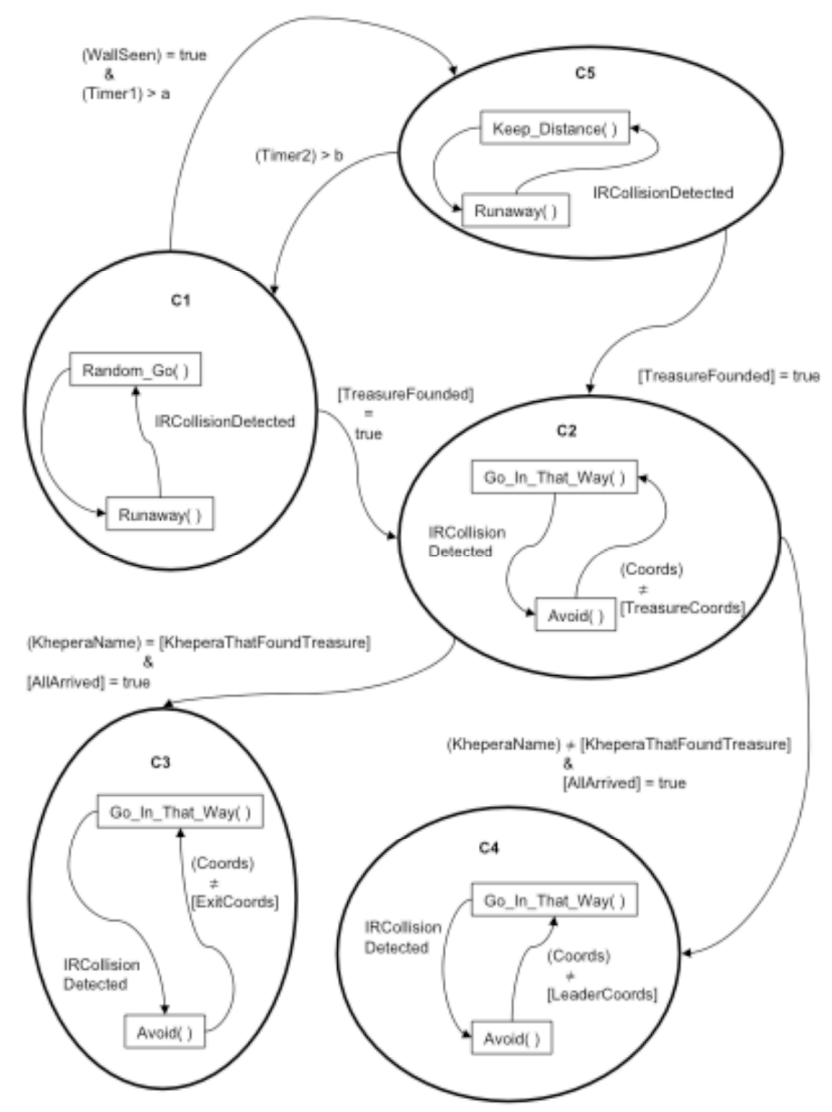

Fig. 9. Hierarchical sets of controllers for virtual agents.

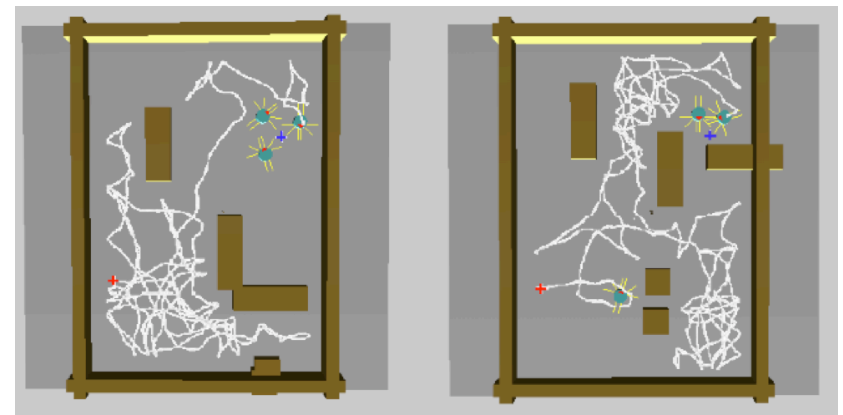

Fig. 10. Results of simulation with virtual agents.

Capitalizing from the results obtained in the previous sections, we have implemented an ad hoc control architecture based on simplified human heuristics. The goal is to provide robots with the ability to explore and search for a target in the environment and to compare their behaviors with that of the human examples. The objective is two-fold (1) to provide a simplified validation of the heuristics extracted from the observation of the human behaviors (considering the limitation due to heterogeneous morphologies and related simplified heuristics) and (2) to endow a robot with an efficient control architecture that should be otherwise hand written with no expert knowledge (e.g. random exploration) or learned (RL or Evolutionary Robotics).

This work was conducted using a modified implementation of the very well known subsomption architecture [2]. This architecture was extended with internal states and subarchitectures. That is, several control architecture implementations co-exist within one robot and only one is active at a time (this is a hierarchical controller). A transition from using one implementation to another depends on internal states and external stimuli (see fig. 9) (e.g. the "exit room" implementation is activated once the target is found). On the other hand, internal states prevents from getting stuck in a dead end behavior (e.g. using clock time out).

The experimental setup consisted of an environment generator that could instantiate always-different environments that nevertheless were part of the same environment class (4-5 obstacles of varying size, random location of the goal/treasure and exit, random starting location, always a possible path towards the goal). We implemented this in the Simbad Autonomous Robot Simulation Package [21] that provides a khepera implementation modeled after the real world Khepera. To stick with the previous experiments with humans, every experiment were conducted with three "blind" khepera using proximity IR sensors and with the ability to detect another close robot, enabling group behavior.

Figure 10 shows two examples of the results obtained. The results can be interpreted from two viewpoints. Firstly, considering performance, the robot succeeded $100 \%$ in all experiments to find the target and retrieve it. This is due to the fact that the hierarchical controller encapsulates sufficiently enough heterogeneous sub-controllers so as to visit all places in the environment. However, no guarantee for optimality exists - it can be seen in the robot traces that the global behavior is far from a patrolling behavior, which should be a 
very good choice (if not the optimal one) when neither the starting point, the map nor the goal location is known.

Secondly, the original goal was not to provide the optimal behavior but rather some validation of the heuristics identified during the human experiments. In this scope, the results obtained with robots share some close relations with that of human subjects. Both follow similar local heuristics (random walk in open space, wall following, time out, etc.) that is not surprising since we implemented this that way and probability of success is always 1 though more optimal behaviors may exist. This $100 \%$ success rate is nevertheless the first and only figure that can be objectively measured, which is not to be underestimated since the success rate for similar tasks is not always guaranteed with other approaches, especially with ad hoc controller. However, psychologist expert analysis on experimental results tend to show that robot behavior are somewhat related to that of their human counterpart (note that these are also sub-optimal behaviors), which while subjective, is what we originally aimed for: experimental validation of an experimental model created after behavior observation and analysis of humans for a similar task and environment.

\section{CONCLUSION AND PERSPECTIVES}

In this paper we addressed the extraction of human heuristics for problem solving in the real world (i.e. finding target in a maze) and the reimplementation of the resulting model in Khepera-like autonomous mobile robots for experimental validation.

We described the whole chain from the experiments and construction of an exhaustive description of the heuristics used towards the implementation using simplified heuristics within a hierarchical control architecture.

The results obtained show that the model obtained can be used for robot controller and lead to an efficient, sub-optimal but human-realistic, behavior for target finding. Moreover a set of techniques to categorize heuristics have been developed in the scope of this work that helped building the heuristicbased behavioral model.

As perspectives, we are already working on a unified experimental setup (see fig. 11). The goal is to control a robot to perform a specific action (finding a target, following a target, patrolling, etc.) - the robot is either controlled by a human or by a program (ad hoc or learned).

Compared to our previous work, the use of one unified setup makes it possible to avoid the gap between the human and the robot perception of the world (it is of course possible to limit perception/action of the human to that of the robot). From the cognitive psychologist viewpoint, there is a strong interest to be able to switch from human control to model validation using the same platform. From the Robotics viewpoint, this makes it possible to address the problem of learning by demonstration in a rather straightforward fashion.

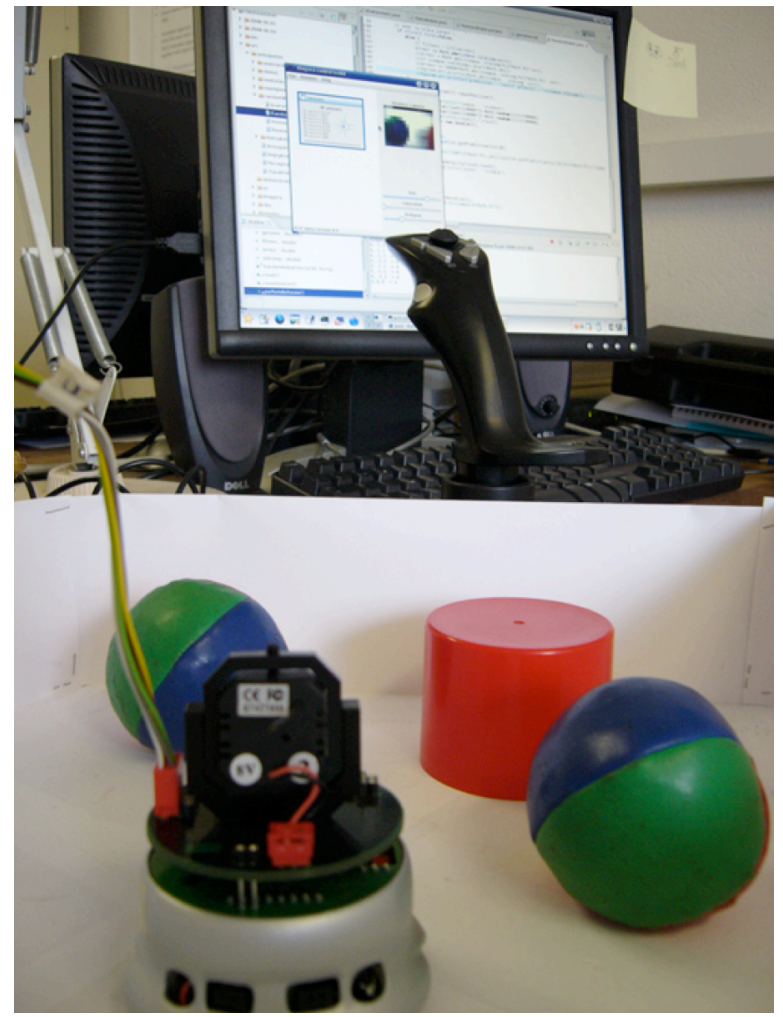

Fig. 11. Learning by demonstration experimental setting. Foreground: Khepera robot, red target, two obstacles. Background: joystick and control screen.

\section{ACKNOWLEDGEMENTS}

We thank Iemmi Valentina, Alexandre Lacaste and Stephan Renaud who participated in the collection of human data.

\section{REFERENCES}

[1] W. Tschacher and C. Scheier. "The perspective of situated and selforganizing cognition in cognitive psychology," In The Nature of Concepts, P. V. Loocke, Ed.,. London: Routledge, 1999, pp. 68-90.

[2] R. A. Brooks, "Intelligence without representation", in Artificial Intelligence, 47, 1991, pp. 139-160.

[3] J. H. Holland, Hidden Order: How Adaptation Builds Complexity. Reading, MA: Perseus Books, 1991.

[4] P. Maes, "Modeling adaptative autonomous agents," in Artificial Life: An Overview, C. G. Langton, Ed., 1997, pp. 135-162.

[5] J. A. Meyer, "The animat approach: simulation of adaptive behavior in animats and robots," in Actes de la conférence neurosciences pour l'ingénieur, F. Alexandre and J. D. Kant, Eds, Publication Loria, 1998, pp. $1-21$.

[6] P.F.M.J. Verschure and P. Althaus "A real-world rational agent: Unifying old and new AI," in Cognitive Science, 2003, 27, 561-590

[7] K. Prepin, Ph., Gaussier, A. Revel and J. Nadel (2005), "A formal approach of developmental robotics and psychology: application to the study of joint attention architecture," in Proceedings Fifth International Workshop on Epigenetic Robotics: Modeling Cognitive Development in Robotic Systems, Nara, Japan, 2005, pp. 97-100,.

[8] C. Tijus, Psychologie Cognitive: une introduction. Paris: Nathan Université, collection Fac, Psychologie, 2005

[9] L. Barsalou, Cognitive Psychology: An Overview for Cognitive Scientists. Hillsdale, NJ: Lawrence Erlbaum, 1992.

[10] Y. LeCun, U. Muller, J. Ben, E. Cosatto, and B. Flepp, "Off-Road Obstacle Avoidance through End-to-End Learning,", in Advances in Neural Information Processing Systems (NIPS), 2005

[11] S. Calinon, F. Guenter, and Aude Billard, A. (2005), "Goal-Directed Imitation in a Humanoid Robot," in Proceedings of the IEEE Intl 
Conference on Robotics and Automation (ICRA), April 18-22, Barcelona, Spain 2005,.

[12] E. Zibetti, and C. Tijus, "Understanding Actions: Contextual Dimensions and Heuristics," in Modeling and Using Context: 5th International and Interdisciplinary Conference on Context, A. Dey, B. Kokinov, D. Leake, R. Turner (Eds). Lecture Notes in Computer Science, 3554, 2005, 542-555

[13] G. Johannson, "Visual perception of biological motion and model of its analysis," in Perception \& Psychophysics, 14, 2005, pp. 201-211.

[14] J.M. Rehg. NSF/DARPA Workshop Perception of Action, Final Report. Prepared by: A. Bobick, MIT Media Lab, 1997.

[15] J.M. Zacks, B. Tversky, \& G. Iyer, "Perceiving, remembering, and communicating structure in events," in Journal of Experimental Psychology-General, 130, 2001, pp. 29-58.

[16] A. Danis, C. Tijus, and A. Santolini, (Accepted for publication), "Interactions cognitives: propriétés, mesures et applications à l'étude des interactions," in I. Olry-Louis, C. Chabrol, and F. Najab, Eds. Interactions communicatives et psychologies: approches actuelles. Paris: Presses de la Sorbonne Nouvelle.

[17] A., Danis, A Santolini, and C. Tijus, "Traitement des Propriétés et Opérationnalisation de la Zone de Développement Proximal," in Psychologie Française, 45, 2000, pp. 165-176.

[18] R.J, Flanagan, and R.S Johansson (2003), "Action plans used in action observation," in Nature, 424, 2003, 769-771.

[19] T. Yamauchi, B. C. Love, and B. Markman, "Learning nonlinearly separable categories by inference and classification," in Journal of Experimental Psychology: Learning, Memory and Cognition, 28, 2002, 585-593.

[20] R. Quinlan (1992). C4.5: Programs for Machine Learning. Morgan Kaufmann Series in Machine Learning, 1992.

[21] L. Hugues, and N. Bredeche. (2006), "Simbad: an Autonomous Robot Simulation Package for Education and Research," in Proceedings of The Ninth International Conference on the Simulation of Adaptive Behavior (SAB'06). Roma, Italy - Springer's Lecture Notes in Computer Sciences / Artificial Intelligence series (LNCS/LNAI) n. 4095, 2006, pp. 831-842. 